\title{
THE ROLE OF MICROWAVE PLASMA TEMPERATURE DURING GRAPHENE NANOSHEETS DEPOSITION ON DIELECTRIC SUBSTRATE: MODELLING AND EXPERIMENT
}

\author{
Martin KUBEČKA, Jozef TOMAN, Miroslav ŠNÍRER, Ondřej JAŠEK, Vít KUDRLE, \\ Jana JURMANOVÁ \\ Department of Physical Electronics, Faculty of Science, Masaryk University, Brno, Czech Republic, EU, \\ kubecka@mail.muni.cz
}

https://doi.org/10.37904/nanocon.2019.8455

\begin{abstract}
The relation between plasma temperature and properties of graphene nanosheet layer deposited on $\mathrm{Si} / \mathrm{SiO}_{2}$ substrate by decomposition of ethanol in microwave plasma torch discharge at atmospheric pressure was investigated in dependence on delivered microwave power and gas flow rates. Plasma modelling was carried out using COMSOL Multiphysics software with delivered microwave power, gas flow rates and experimental reactor geometry as input parameters. Results of the heat flow and fluid dynamics modelling were compared with substrate temperature measured by thermocouple integrated in quartz tube substrate holder. The graphene nanosheets layer was characterized by SEM, Raman spectroscopy and 4-point probe method. The layers were severals tens of $\mu \mathrm{m}$ thick and their sheet resistance varied from 2 to $40 \mathrm{k} \Omega / \mathrm{sq}$. The properties of individual graphene nanosheets, $2 \mathrm{D} / \mathrm{G}$ and $\mathrm{D} / \mathrm{G}$ Raman band ratio, as well as the sheet resistance of their conductive network were correlated with the increase of plasma temperature with increasing microwave power. The substrate temperature increased linearly with delivered microwave power and the layer sheet resistance was decreasing with increasing microwave power and saturated at $2 \mathrm{k} \Omega / \mathrm{sq}$ and $\mathrm{D} / \mathrm{G}$ ratio of 0.6 .
\end{abstract}

Keywords: Graphene, microwave plasma, temperature, dielectric substrate

\section{INTRODUCTION}

Microwave plasma decomposition of ethanol is single-step, environmentally friendly way of producing graphene in the form of powder. This method was first published by Dato [1] and further investigated by Tatarova and Tsyganov [2]. It was shown that ethanol and dimethyl ether have the ideal ratio of $\mathrm{C}, \mathrm{H}$ and $\mathrm{O}$ atoms for controllable graphene synthesis. Tsyganov's theoretical model of this synthesis process could simulate and experimentally map the particle and thermal fluxes in the plasma reactor. The model is based on a set of nonlinear differential equations describing plasma thermodynamics and chemical kinetics. The model showed that high temperature atmospheric microwave plasma, with temperatures $4000 \mathrm{~K}$ and higher, leads to formation of $\mathrm{CO}$ and $\mathrm{H}_{2}$, and assembly of solid product from acetylene molecules which get decomposed to $\mathrm{C}$ and $\mathrm{H}$ atoms. $\mathrm{C}_{2}$ molecule was determined to be the main plasma species responsible for graphene growth. Recently, Bundaleska et al. showed that use of methane with appropriate plasma conditions (gas flows, power) can lead to formation of graphene nanosheets as well [3]. Similarly carbon nanotubes and graphene nanosheets could be prepared in TIAGO torch by group of Rincon and Melero without use of catalyst [3], [4]. We have recently studied synthesis of graphene nanostructures in dependence on plasma conditions using dual-channel microwave plasma torch at atmospheric pressure [5] and showed that plasma dynamics and plasma properties (temperature, carbon species concentration) strongly influences prepared material. We have also showed that depending on deposition conditions carbon nanotube layer with height up to $200 \mu \mathrm{m}$ could be prepared directly on the dielectric substrate using Fe catalyst [6].

In our work we couple gas dynamics and heat transfer model of our reactor with substrate temperature and show that electrical conductivity of graphene nanosheets layer deposited on dielectric substrate is correlated with intrinsic plasma properties. 


\section{EXPERIMENTAL}

The graphene nanosheets were synthesized by ethanol decomposition in microwave plasma torch at atmospheric pressure. The microwave discharge was ignited inside reactor formed by quartz tube ( $80 \mathrm{~mm}$ diameter, $200 \mathrm{~mm}$ length) terminated by dural flanges. The discharge electrode was hollow nozzle (Figure 1) with central channel used for introduction of working gas - argon (360 - $920 \mathrm{sccm})$ and subsequent ignition of plasma and secondary channel (annulus with outer radius $8.4 \mathrm{~mm}$ and inner radius $7.7 \mathrm{~mm}$ ) was used for introduction of carrying gas - argon (500 - $1400 \mathrm{sccm}$ ) with precursor (ethanol 2-25 sccm) vapours into the plasma environment. Synthesized nanopowder was collected from the reactor wall or on the $\mathrm{Si} / \mathrm{SiO}_{2}(92 \mathrm{~nm})$ substrates fixed in the holder Figure 2. More details about the experimental setup can be found in [5]. Raman spectroscopy was carried out using HORIBA LabRAM HR Evolution system with $532 \mathrm{~nm}$ laser, using 100x objective and $25 \%$ ND filter in the range from 1000 to $3200 \mathrm{~cm}^{-1}$. Samples were imaged with TESCAN scanning electron microscope (SEM) MIRA3 with Schottky field emission electron gun equipped with secondary electron (SE) and back-scattered electron (BSE) detectors as well as Oxford Instruments EDX analyser. Sheet resistance was measured by homemade 4-point probe system, probe distance d $1.06 \mathrm{~mm}$, equipped with Keithley Keysight 34465 multimeter. Substrate temperature was measured by $\mathrm{K}$ thermocouple with compensation line JUMO 901250/32-1043-1.5-300-48-2500. Substrates used were $15 \times 15 \mathrm{~mm}$ one-side polished Si (100) P type, $525 \mathrm{~mm}$ thickness wafers from ON Semiconductor s.r.o. (Czech Republic) with $92 \mathrm{~nm}$ thermally oxidized $\mathrm{SiO}_{2}$. Ethanol p.a. 99.8\% from Penta was purchased from Verkon (Czech Republic). Pure gas, argon 99.998\%, was purchased from Messer Technogas, s.r.o. (Czech Republic).
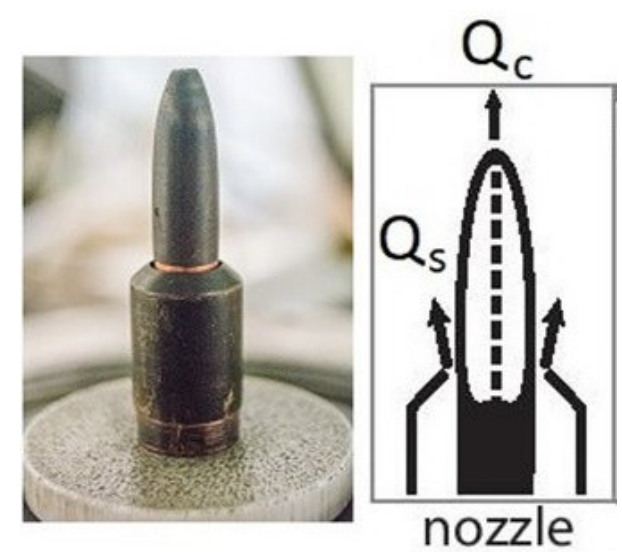

Figure 1 Plasma nozzle scheme

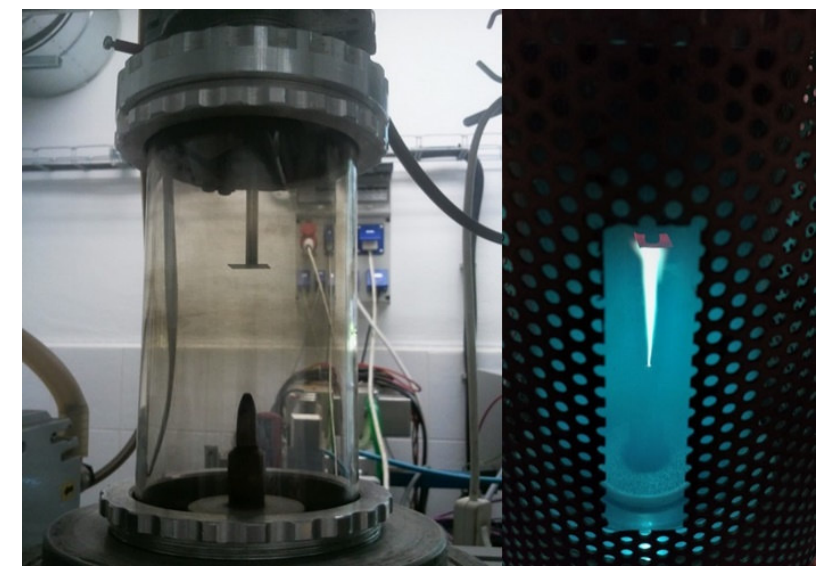

Figure 2 Substrate holder and sample heating during deposition

\section{DESCRIPTION OF THE MODEL}

We employed a coupled model between gas dynamics and heat transfer. The model was solved on unstructured mesh grid using COMSOL Multiphysics based on the finite element method. The geometry of the simulated reactor was approximated by $2 \mathrm{D}$ axially symmetric domain. The steady state turbulent fluid flow was described by the Reynolds-averaged Navier-Stokes equations (RANS) and describes the gas dynamics and mixing of argon and ethanol. The heat equation describing the neutral gas consider the heat source due to the elastic collision with electrons as well as endothermic and exothermic reactions in the plasma. The temperature field was validated with experimental measurements to further refine of the simulation results.

\section{RESULTS AND DISCUSSION}

Microwave plasma temperature strongly influenced deposition process of graphene nanosheets in microwave plasma torch discharge at atmospheric pressure. While plasma dynamics was mainly influenced by carried gas and precursor flow rate [5], the plasma rotational temperature - neutral gas temperature was mainly 
influenced by delivered microwave power ( $\mathrm{Pw}$ ) Figure 3. This dependance then caused dependence of the substrate temperature on delivered microwave power Figure 4.

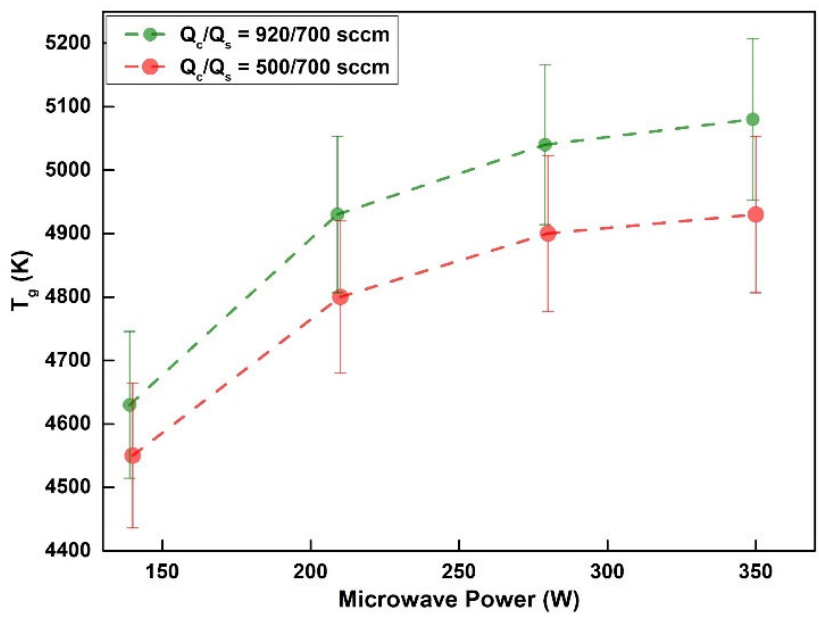

Figure 3 Dependance of plasma temperature on microwave power $\mathrm{Pw}$

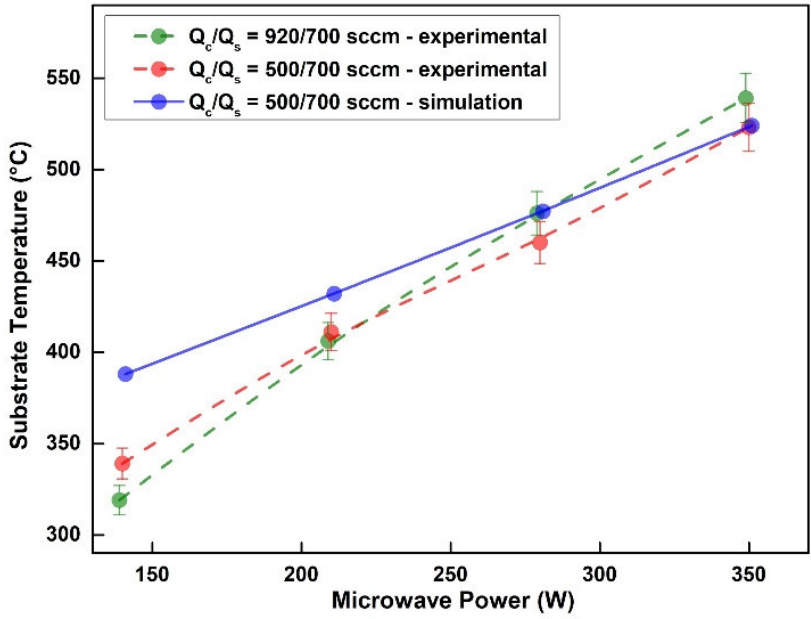

Figure 4 Substrate temperature comparison of model and measurement

This dependence was compared with the result of our plasma model in COMSOL Multiphysics software. First we investigated the influence of gas flow rate on gas dynamics in our reactor and position of substrate in the discharge axis i. e. distance from the plasma nozzle Figure 5. As can be seen the introduction of substrate into reactor disturbed the laminar gas flow and in consequence also formation of graphene nanosheets conductive network on the substrate. This effect was further amplified by heat flow in the plasma reactor and changed the shape of the discharge with increasing the gas velocity in the vicinity of substrate. The geometry of the reactor also caused gas recirculation as can be seen in Figure 5.
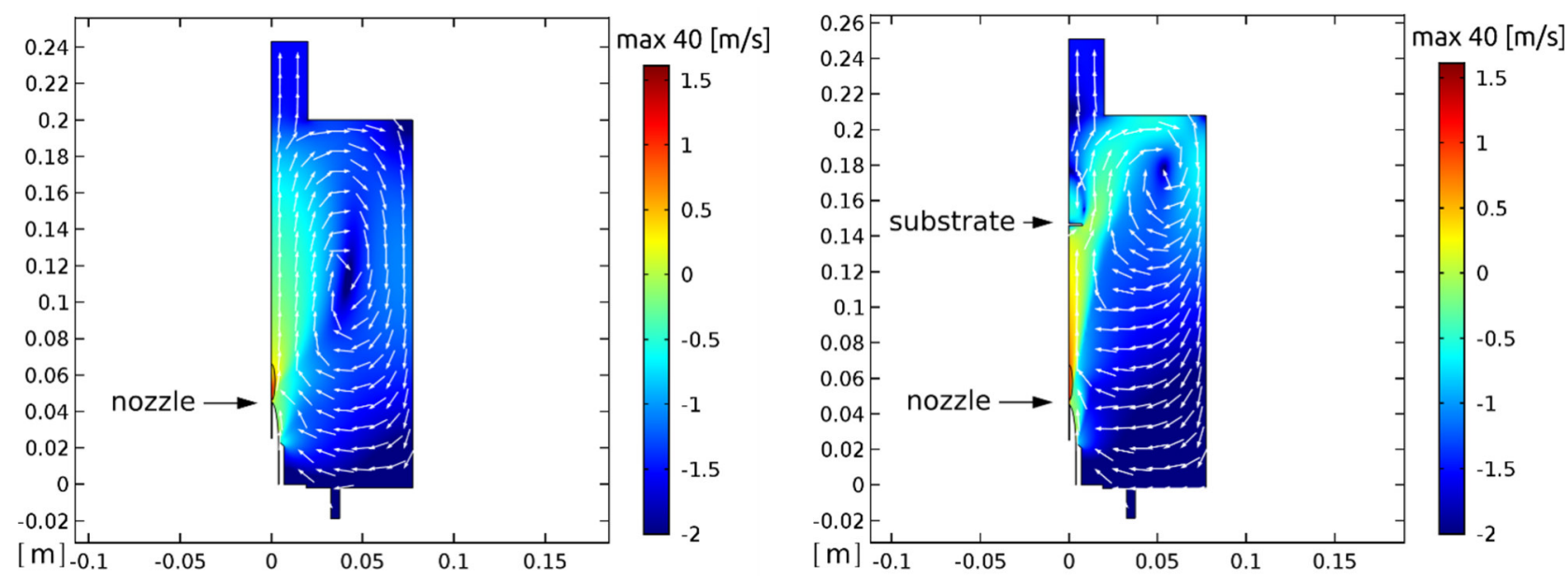

Figure 5 Velocity of the gas flow with normalized velocity field in log scale in the chamber without the substrate (left) and with the substrate (right)

We compared the temperatures measured by the thermocouple in contact with the substrate with results of the model (Figure 4) and the obtained values were in good agreement except the lowest Pw. This difference was caused by contraction of the discharge length which increased the distance between the discharge and the substrate and led to decrease of the substrate temperature during the experiment. Placing the substrate in an appropriate distance and choosing correct deposition conditions (Qc/Qs flow rate) resulted in 
homogenous deposition of graphene nanosheets with given properties, 2D/G and D/G Raman band ratio [7]. Such a deposition led to the formation of conductive carbon network on dielectric substrate.

We used $15 \times 15 \mathrm{~mm} \mathrm{Si} / \mathrm{SiO}_{2}$ wafers placed $10 \mathrm{~cm}$ from the plasma nozzle to deposit graphene nanosheet layer. During the deposition, the deposition time was 5 minutes, whole substrate was covered with graphene nanosheets layer with thickness ( $\mathrm{t}$ ) varying from 10 to $40 \mu \mathrm{m}$ and small variation of thickness along the sample edges. Giving the thickness of the layer being much smaller than sample size $(t<l)$ and probe distance $(t<d)$, we used sheet resistance Rsq [ $\Omega / s q]$ values for electrical characterization of the layers [8]. The substrate temperature was dependent on the delivered plasma power and was $300^{\circ} \mathrm{C}$ and $550{ }^{\circ} \mathrm{C}$ for $140 \mathrm{~W}$ and $350 \mathrm{~W}$, respectively. It is also possible to regulate the substrate temperature by changing the distance between the plasma and the substrate, but it was not used in this work. To ensure that the substrate temperature was not influencing deposited material, we compared the material collected on the reactor wall and the substrate and the results of the Raman spectroscopy agree with each other. The results of the measurement are summarized in Figure 6 and Figure 7, which showed the relation between the nanosheet layer resistivity and delivered microwave power. As can be seen the same correlation was observed with neutral gas temperature during the decomposition of ethanol and also with $\mathrm{C}_{2} / \mathrm{C}$ species ratio determined by optical emission spectroscopy.

Conductive carbon layers play an important role in possible future applications such as flexible electronics and displays [9] or sensors [10]. In our case the conductive network of graphene nanosheets was created only in the case of thicker, several $\mu \mathrm{m}$, layer of deposited material. Such layer was not suitable for transparent electrode application but could be used for various gas and electrochemical sensors. The sheet resistance of the graphene nanosheet layer varied from 2 to $40 \mathrm{k} \Omega / \mathrm{sq}$ depending on the quality of synthesized graphene nanosheets. The sheet resistance decreased with increasing 2D/G peak ratio and decreasing D/G peak ratio and increasing $\mathrm{sp}^{2}$ content in prepared nanostructures i.e. increase of plasma power. This can be understood in terms of presence of higher amount of defects in the nanosheet structure (high intensity of Raman D band and higher $\mathrm{sp}^{3}$ content) which caused higher resistance for electrical current flow through the nanosheet layer. This dependence saturates around D/G of 0.6 and Rsq of $2 \mathrm{k} \Omega / \mathrm{sq}$ where influence of conductive network structure exceeded that of nanosheets.

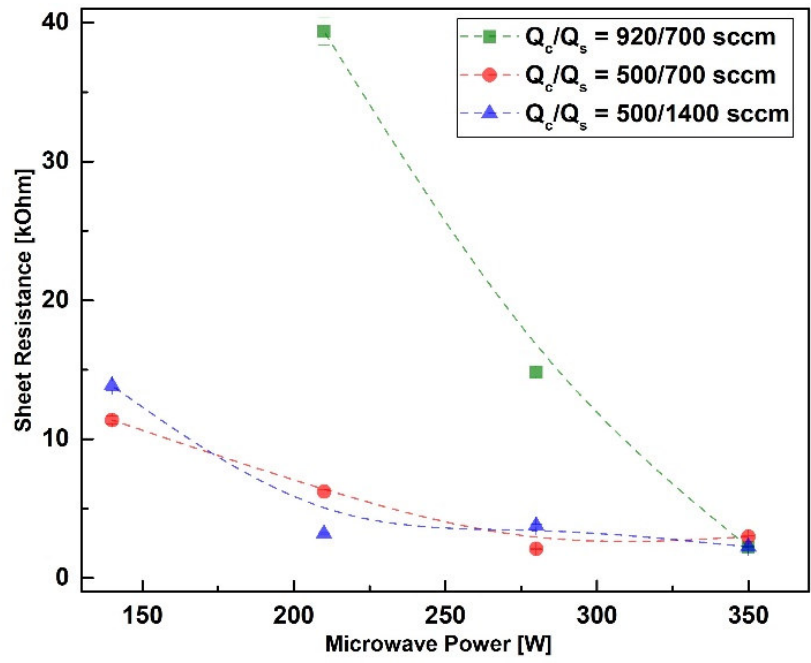

Figure 6 Sheet resistance dependence on Qc/Qs and Pw

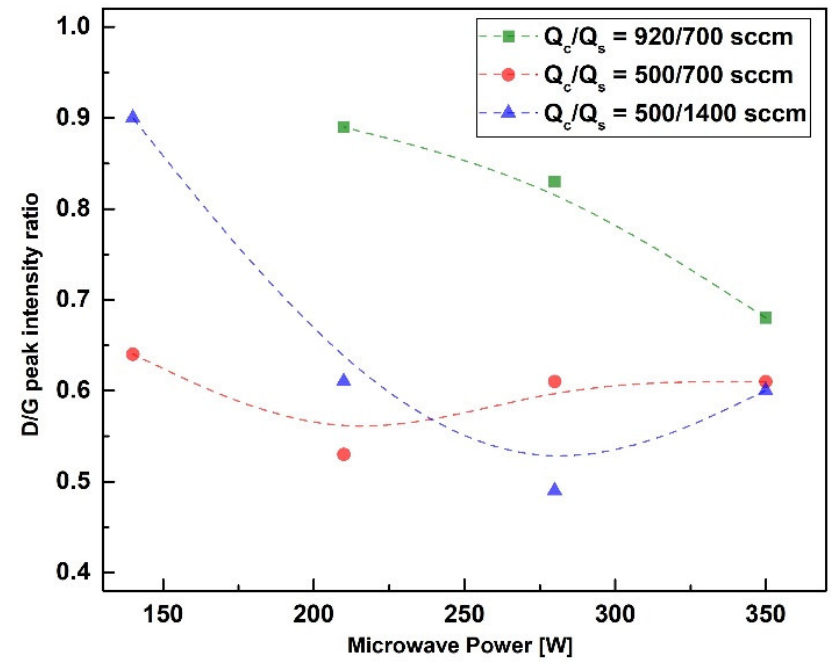

Figure 7 D/G Raman band ratio dependence on $\mathrm{Pw}$

\section{CONCLUSION}

The layers of carbon nanosheets were successfully deposited on the $\mathrm{Si}_{/} \mathrm{SiO}_{2}$ substrate in microwave plasma torch discharge without influencing the synthesis process. The sheet resistance of such prepared layers 
decreased, from 40 to $2 \mathrm{k} \Omega / \mathrm{sq}$, with increase of microwave power i.e. lower amount of defects in the nanosheet structure and increasing substrate temperature. The modelling of gas dynamics and heat transfer in the discharge reactor showed strong influence of the substrate on the velocity of the gas in the vicinity of the substrate. The observed increase of substrate temperature with increased delivered microwave power was in good agreement with discharge model.

\section{ACKNOWLEDGEMENTS}

This work was supported by The Czech Science Foundation (GA CR) under project 18-08520S and in part by the project LO1411 (NPU I) funded by Ministry of Education, Youth and Sports of Czech Republic. We would like to thank

\section{REFERENCES}

[1] A. DATO, V. RADMILOVIC, Z. LEE, J. PHILLIPS, and M. FRENKLACH, "Substrate-free gas-phase synthesis of graphene sheets," Nano Lett., 2008, vol. 8, no. 7, pp. 2012-2016.

[2] D. TSYGANOV et al., "On the plasma-based growth of 'flowing' graphene sheets at atmospheric pressure conditions," Plasma Sources Science and Technology, 2016, vol. 25, no. 1. p. 015013.

[3] N. BUNDALESKA et al., "Microwave plasma enabled synthesis of free standing carbon nanostructures at atmospheric pressure conditions," Phys. Chem. Chem. Phys., 2018, vol. 20, no. 20, pp. 13810-13824.

[4] R. RINCÓN, C. MELERO, M. JIMÉNEZ, and M. D. CALZADA, "Synthesis of multi-layer graphene and multi-wall carbon nanotubes from direct decomposition of ethanol by microwave plasma without using metal catalysts," Plasma Sources Science and Technology, 2015, vol. 24, no. 3. p. 032005.

[5] J. TOMAN, O. JASEK, M. SNIRER, V. KUDRLE, and J. JURMANOVA, "On the interplay between plasma discharge instability and formation of free-standing graphene nanosheets in a dual-channel microwave plasma torch at atmospheric pressure," Journal of Physics D: Applied Physics, 2019, vol. 52, no. 26. p. 265205.

[6] O. JAŠEK et al., "Discussion of important factors in deposition of carbon nanotubes by atmospheric pressure microwave plasma torch," Journal of Physics and Chemistry of Solids, 2007, vol. 68, no. 5-6. pp. 738-743.

[7] A. C. FERRARI, "Raman spectroscopy of graphene and graphite: Disorder, electron-phonon coupling, doping and nonadiabatic effects," Solid State Communications, 2007, vol. 143, no. 1-2. pp. 47-57.

[8] I. MICCOLI, F. EDLER, H. PFNÜR, and C. TEGENKAMP, "The 100th anniversary of the four-point probe technique: the role of probe geometries in isotropic and anisotropic systems," J. Phys. Condens. Matter, 2015, vol. 27, no. 22, p. 223201.

[9] H. JANG, Y. J. PARK, X. CHEN, T. DAS, M.-S. KIM, and J.-H. AHN, "Graphene-Based Flexible and Stretchable Electronics," Adv. Mater., 2016, vol. 28, no. 22, pp. 4184-4202.

[10] C. I. L. JUSTINO, A. R. GOMES, A. C. FREITAS, A. C. DUARTE and T. A. P. ROCHA-SANTOS, "Graphene based sensors and biosensors," TrAC Trends in Analytical Chemistry, 2017, vol. 91. pp. 53-66. 